\title{
OPEN Rapid high-resolution melting genotyping scheme for Escherichia coli based on MLST derived single nucleotide polymorphisms
}

Matej Bezdicek ${ }^{1,2}$, Marketa Nykrynova ${ }^{3}$, Karel Sedlar $^{3}$, Stanislava Kralova ${ }^{5}$, Jana Hanslikova ${ }^{1}$ Aja Komprdova ${ }^{1}$, Helena Skutkova ${ }^{3}$, Iva Kocmanova ${ }^{4}$, Jiri Mayer ${ }^{1}$ \& Martina Lengerova ${ }^{1,2 \rrbracket}$

Routinely used typing methods including MLST, rep-PCR and whole genome sequencing (WGS) are time-consuming, costly, and often low throughput. Here, we describe a novel mini-MLST scheme for Eschericha coli as an alternative method for rapid genotyping. Using the proposed mini-MLST scheme, 10,946 existing STs were converted into 1,038 Melting Types (MelTs). To validate the new mini-MLST scheme, in silico analysis was performed on 73,704 strains retrieved from EnteroBase resulting in discriminatory power $\mathrm{D}=0.9465$ ( $\mathrm{Cl} 95 \% 0.9726-0.9736)$ for mini-MLST and $\mathrm{D}=0.9731$ (CI 95\% 0.9726-0.9736) for MLST. Moreover, validation on clinical isolates was conducted with a significant concordance between MLST, rep-PCR and WGS. To conclude, the great portability, efficient processing, cost-effectiveness, and high throughput of mini-MLST represents immense benefits, even when accompanied with a slightly lower discriminatory power than other typing methods. This study proved mini-MLST is an ideal method to screen and subgroup large sets of isolates and/or quick strain typing during outbreaks. In addition, our results clearly showed its suitability for prospective surveillance monitoring of emergent and high-risk $E$. coli clones'.

Escherichia coli is a gram-negative bacteria commonly colonizing the human gastrointestinal tract as a harmless commensal microorganism. However, it is also one of the major human pathogens causing mainly urinary tract infections, bloodstream infections, neonatal sepses, skin structure infections and traveller's diarrhea ${ }^{1}$. Together with the globally increasing rates of antibiotic resistance including E. coli strains, extraintestinal infections caused by this opportunistic pathogen represent one of the main public health problems.

Bacterial genotyping is a powerful tool for strain identification, outbreak investigation and surveillance applications. Electrophoretic techniques such as pulse-field gel electrophoresis (PFGE) or repetitive element sequence-based PCR (rep-PCR) have been considered the gold standard methods for bacterial strain tracking for many years ${ }^{2}$. Although PFGE is a highly discriminatory and robust method, its disadvantages such as extreme time and labor demands combined with potential resolution issues and inconsistencies in interpretation make it difficult to standardize between laboratories. In contrast, sequence-based typing such as multilocus sequence typing (MLST) provides a robust, portable, interlaboratory system based on sequencing 6-8 housekeeping genes ${ }^{3}$. Despite its costs and demands on time, MLST has become a typing technique used worldwide to track the spread of virulent and antibiotic-resistant strains on a local and global level. Recently, whole genome sequencing (WGS) has been introduced and has become a widely used sequence-based genotyping tool. Along with the rapid development, WGS technology has also become more accessible for routine applications. However, it is still impractical for prospective typing and/or screening typing larger numbers of strains. In particular, data processing and their evaluation are currently WGS' main disadvantage, demanding both extra computational and human resources.

Another method that can be used for genotyping is high-resolution melting (HRM). HRM is a highly specific and sensitive method to discriminate variants in PCR amplicons ${ }^{4}$. It is based on dsDNA's melting separation with monitoring via changes in fluorescence intensity, which correlates with the CG content and amplicon length.

${ }^{1}$ Department of Internal Medicine, Hematology and Oncology, University Hospital Brno, Brno, Czech Republic. ${ }^{2}$ Department of Internal Medicine, Hematology and Oncology, Masaryk University, Brno, Czech Republic. ${ }^{3}$ Department of Biomedical Engineering, Faculty of Electrical Engineering and Communication, Brno University of Technology, Brno, Czech Republic. ${ }^{4}$ Department of Clinical Microbiology and Immunology, University Hospital Brno, Brno, Czech Republic. ${ }^{5}$ Department of Experimental Biology, Czech Collection of Microorganisms, Faculty of Science, Masaryk University, Brno, Czech Republic. ${ }^{\circledR}$ email: Lengerova.Martina@fnbrno.cz 


\begin{tabular}{|c|c|c|c|c|c|}
\hline Gene & Target region & Primer ID & Primer sequence $5^{\prime}-3^{\prime}$ & $\begin{array}{l}\text { Amplicon length } \\
\text { (bp) }\end{array}$ & $\begin{array}{l}\text { Predicted alleles (based } \\
\text { on GC content) }\end{array}$ \\
\hline \multirow[t]{2}{*}{$a d k$} & $288-368$ & adk322 FW & \begin{tabular}{|l|} 
GGCATCAATGTT \\
GATTACGTTC
\end{tabular} & \multirow{2}{*}{122} & \multirow{2}{*}{$43, \mathbf{4 4}, \mathbf{4 5}, \mathbf{4 6}, \mathbf{4 7}, 48,49,52$} \\
\hline & & adk322 RV & $\begin{array}{l}\text { GGCGGATTGAAT } \\
\text { TTAACGT }\end{array}$ & & \\
\hline \multirow[t]{2}{*}{ fum $C$} & $227-390$ & fumC327 FW & $\begin{array}{l}\text { CTGCGCAAGCAA } \\
\text { CTCATTC }\end{array}$ & \multirow{2}{*}{152} & \multirow{2}{*}{$\begin{array}{l}50,51,52,55,56,57, \mathbf{5 8}, 59 \\
60, \mathbf{6 1}, \mathbf{6 2}, \mathbf{6 3}, 64,65\end{array}$} \\
\hline & & fumC327 RV & $\begin{array}{l}\text { GCTACCCAGCCG } \\
\text { GAAATCT }\end{array}$ & & \\
\hline \multirow[t]{2}{*}{$\operatorname{gyr} B$} & $147-191$ & gyrB174 FW & $\begin{array}{l}\text { GTTACCGGCGAG } \\
\text { ACTGAAAA } \\
\end{array}$ & \multirow{2}{*}{85} & \multirow{2}{*}{$22,23, \mathbf{2 4}, \mathbf{2 5}, \mathbf{2 6}, \mathbf{2 7}, 28$} \\
\hline & & gyrB174 RV & $\begin{array}{l}\text { ATTCGAACTCGG } \\
\text { TCACATTG }\end{array}$ & & \\
\hline \multirow[t]{2}{*}{$i c d$} & $144-218$ & icd174 FW & $\begin{array}{l}\text { TCTGATTCGTGA } \\
\text { ATATCGCG }\end{array}$ & \multirow{2}{*}{116} & \multirow{2}{*}{$41,42, \mathbf{4 3}, \mathbf{4 4}, \mathbf{4 5}, 46, \mathbf{4 7}, 48$} \\
\hline & & icd174 RV & $\begin{array}{l}\text { GCAGGCAGATGT } \\
\text { AGAGATCCA }\end{array}$ & & \\
\hline \multirow[t]{2}{*}{ purA } & $254-334$ & purA263 FW & $\begin{array}{l}\text { CTGTTGCCGACA } \\
\text { TCCTGAC }\end{array}$ & \multirow{2}{*}{120} & \multirow{2}{*}{$43, \mathbf{4 4}, \mathbf{4 5}, \mathbf{4 6}, \mathbf{4 7}, 48,53$} \\
\hline & & purA263 RV & $\begin{array}{l}\text { GGTCGATATCCA } \\
\text { GCAGCGTA }\end{array}$ & & \\
\hline \multirow[t]{2}{*}{$\operatorname{rec} A$} & $82-143$ & recA91 FW & $\begin{array}{l}\text { CAGGCACTGGAA } \\
\text { ATCTGTGA }\end{array}$ & \multirow{2}{*}{100} & \multirow{2}{*}{$\begin{array}{l}34, \mathbf{3 8}, \mathbf{3 9}, \mathbf{4 0}, \mathbf{4 1}, 42,43 \\
44,45,46\end{array}$} \\
\hline & & recA91 RV & $\begin{array}{l}\text { CTTCGATTTCCG } \\
\text { CTTTCG }\end{array}$ & & \\
\hline
\end{tabular}

Table 1. Target SNP positions, primer information and predicted melting alleles for mini-MLST scheme. Allele prediction is based on the total number of GC bases between primers in the targeted region. Alleles captured in our sample set are shown in bold.

Bacterial strain typing using HRM detection of single nucleotide polymorphisms (SNPs), called mini-MLST or minim typing, has already been successfully applied to Klebsiella pneumoniae ${ }^{5}$, Staphylococcus aureus ${ }^{6}$, Enterococcus faecium $^{7}$ and Streptococcus pyogenes ${ }^{8}$. These methods are based on detecting allelic specific SNPs derived from well-established MLST schemes used worldwide. Regarding E. coli, methods based on HRM were already used for multiple purposes such as species identification ${ }^{9}$, detecting and quantifying of enterotoxigenic virulence factors ${ }^{10}$, detecting of AmpC, ESBL and carbapenemase genes ${ }^{11}$ and in combination with ligation-mediated realtime PCR for molecular typing on a local level ${ }^{12}$.

Here we describe a novel mini-MLST scheme for E. coli as an alternative method for rapid genotyping suitable for routine clinical practice. We compared the proposed mini-MLST with commonly used molecular typing such as REP-PCR and MLST on an outbreak of E. coli at the Neonatal Department at the University Hospital Brno (UHB). In addition, our novel mini-MLST scheme was also compared to WGS on E. coli strains collected during a surveillance study at the Department of Hematology and Oncology (UHB).

\section{Results}

Method design. Firstly, all SNPs in the E. coli MLST ${ }^{13}$ loci were identified using Minimum SNPs software ${ }^{14}$. Consequently, the SNPs that do not represent a nucleotide exchange affecting the number of hydrogen bonds $(\mathrm{A} / \mathrm{T} \leftrightarrow \mathrm{G} / \mathrm{C})$ were excluded, because these are not commonly detectable by HRM. All SNPs without flanking conserved regions were also excluded from future analyses as those are crucial for primer design. Simpson's Index of Diversity (D) was calculated. For all the remaining SNPs. Finally, a set of six regions containing highly informative SNP sets meeting our criteria was found in 6 different MLST loci. The regions were labelled as (locus name + first and last position of region of interest) adk288-368, fumC227-390, gyrB147-191, icd144-218, purA254-334 and recA82-143 (Table 1). No D-optimized SNPs in the $m d h$ could be found to meet our miniMLST design criteria. The amplified fragments sizes ranged between 85 to $152 \mathrm{bp}$. The total number of alleles $(\mathrm{n})$ and $\mathrm{D}$ values were calculated to determine the discriminatory power of each loci containing the targeted SNPs as follows: $a d k 288-368(\mathrm{n}=161, \mathrm{D}=0.8784$, CI 95\% 0.8657-0.8911), fumC227-390 ( $\mathrm{n}=456, \mathrm{D}=0.9605$, CI 95\% 0.9561-0.9649), gyrB147-191 ( $\mathrm{n}=81, \mathrm{D}=0.7380$, CI 95\% 0.7111-0.7649), icd144-218 ( $\mathrm{n}=179, \mathrm{D}=0.8664$, CI 95\% 0.8536-0.8793), purA254-334 $(\mathrm{n}=144, \mathrm{D}=0.8182$, CI 95\% 0.7956-0.8408) and recA82-143 $(\mathrm{n}=131$, $\mathrm{D}=0.7175$, CI 95\% 0.6829-0.7520).

We used our in-house MLST2MELT software to predict mini-MLST alleles for all 6 regions (Table 1) and generate a MelT key to work as a conversion key between STs and MelTs. In 3/2021, 10,946 existing STs were converted into 1,038 MelTs. Out of a total of 10,946 STs, 39 STs were not assigned to a MelT. ST1942 and ST339's MLST schemes contain alleles missing in the source database. Additionally, the following alleles carry a high polymorphism or deletion content in the primer binding region: adk 44; fum C 42, 255, 256, 624, 683, 684, 808, $809,1012,1021,1030,1050,1216,1273,1594,1664 ;$ gyrB 212, 213, 311, 331,354, 427, 428, 741, 747, 1024, icd 40, 1306 and purA 99, 428. In the Melt Key, STs containing these alleles are assigned to the MelT0 and appended 
adk288-368

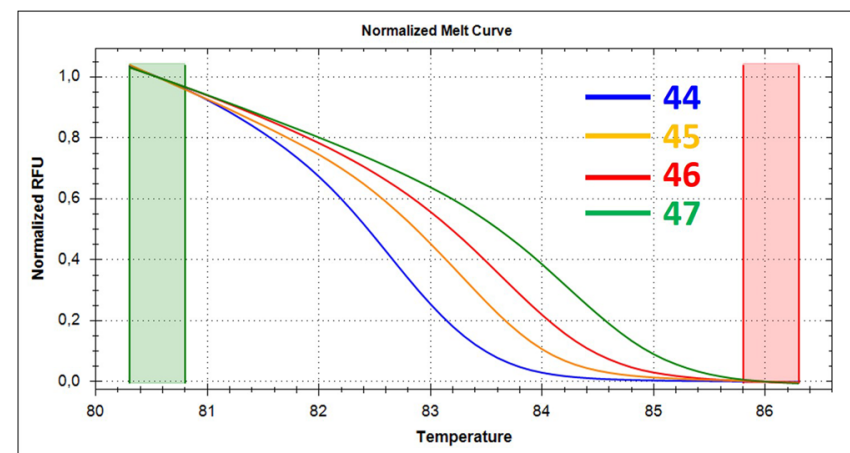

gyrB147-191

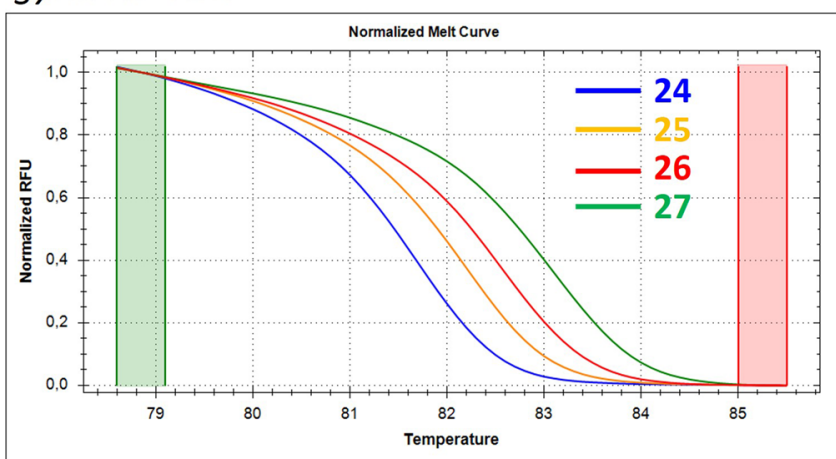

purA254-334

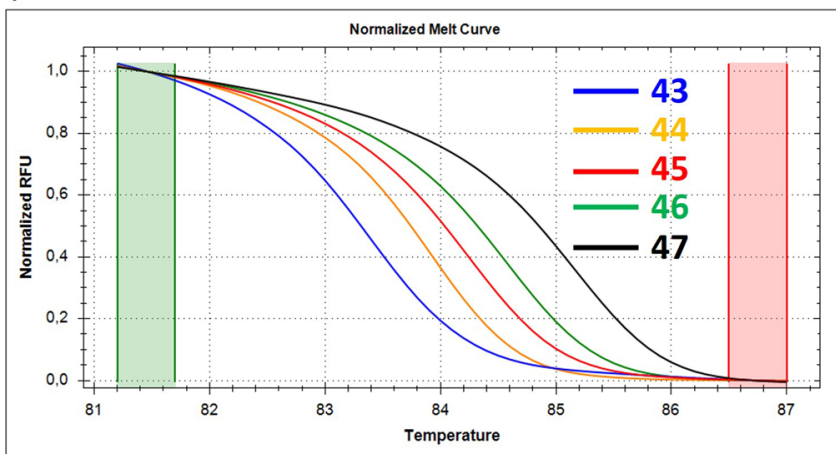

fumC227-390

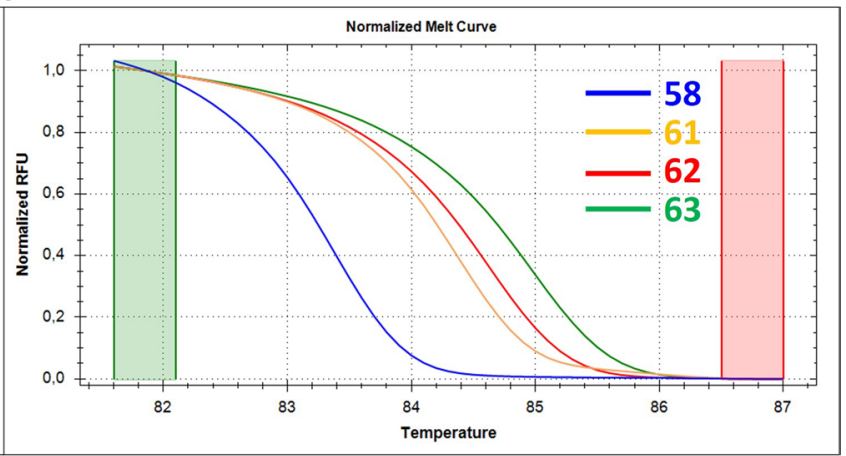

icd144-218

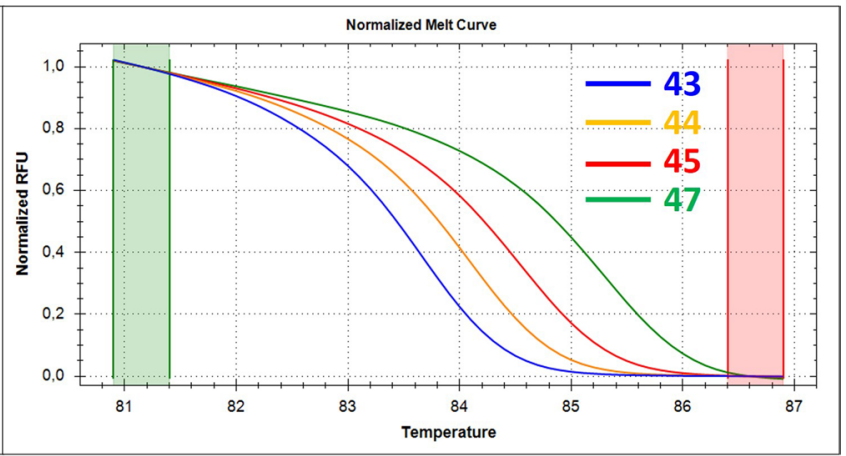

recA82-143

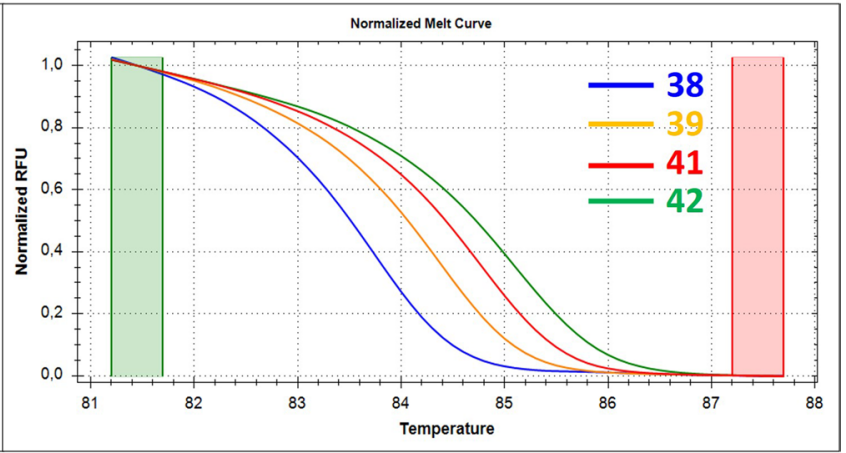

Figure 1. High-resolution melting curves for each of six mini-MLST loci. The curves are labeled with the GC content in corresponding mini-MLST loci. For locus adk288-368, four $(44,45,46,47)$ out of eight predicted alleles are displayed. For locus fum $\mathrm{C} 227-390$, four $(58,61,62,63)$ out of 14 predicted alleles are displayed. For

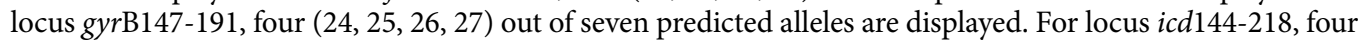
$(43,44,45,47)$ out of eight predicted alleles are displayed. For locus purA254-334, five $(43,44,45,46,47)$ out of seven predicted alleles are displayed. For locus recA82-143, four $(38,39,40,41)$ out of 10 predicted alleles are displayed.

with a note indicating that the certain region may fail to amplify, or the ST may carry an allele missing from the MLST database.

Method validation. The HRM curves for each mini-MLST loci were obtained for all 169 E. coli isolates and are presented in Fig. 1. The corresponding difference curves are shown in Fig. 2. The corresponding melting peaks can be found in Supplementary Fig. S1. Representatives from each obtained HRM curve were sequenced using MLST primers to determine the GC content in specific mini-MLST loci. The GC values were subsequently used to identify mini-MLST alleles. The melting temperature (Tm) values for each mini-MLST allele were calculated (Table 2). The HRM curves from four isolates had a non-standard shape and differed from the remaining HRM curves in at least one mini-MLST loci (an example of non-standard HRM curves can be found in Supplementary Fig. S2). Using Sanger sequencing, we found these isolates to be a mixture of at least two different strains and they were therefore excluded from further analyses.

MelTs were assigned to each isolate based on the acquired HRM curves and our MelT conversion key. From 165 isolates, 34 different MelTs were determined (Table 3). To correlate MelTs and STs, a subgroup of 110 isolates including at least one isolate of each obtained MelT was subjected to complete MLST. Those 110 isolates 
adk288-368

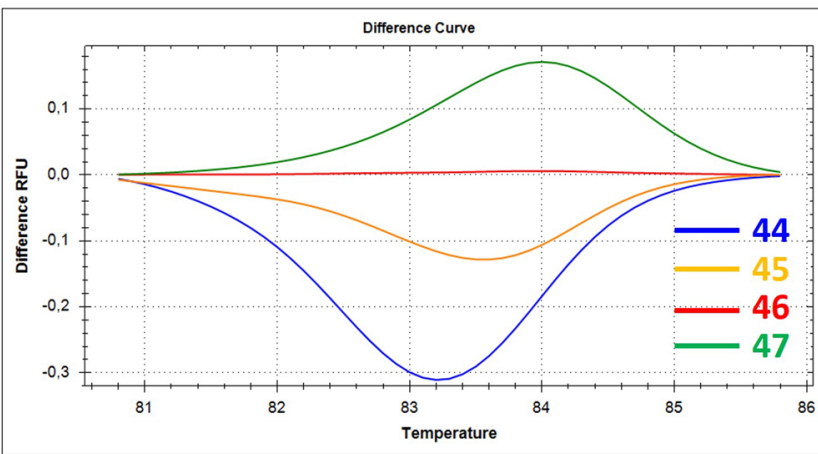

gyrB147-191
fumC227-390

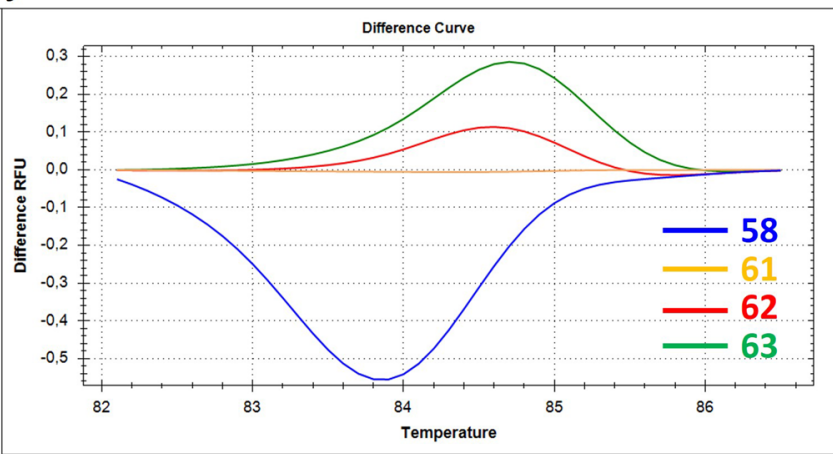

icd144-218

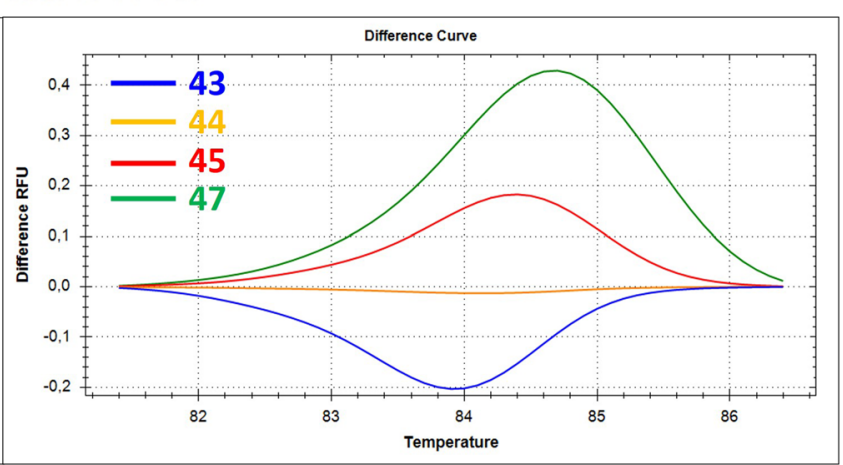

recA82-143

purA254-334

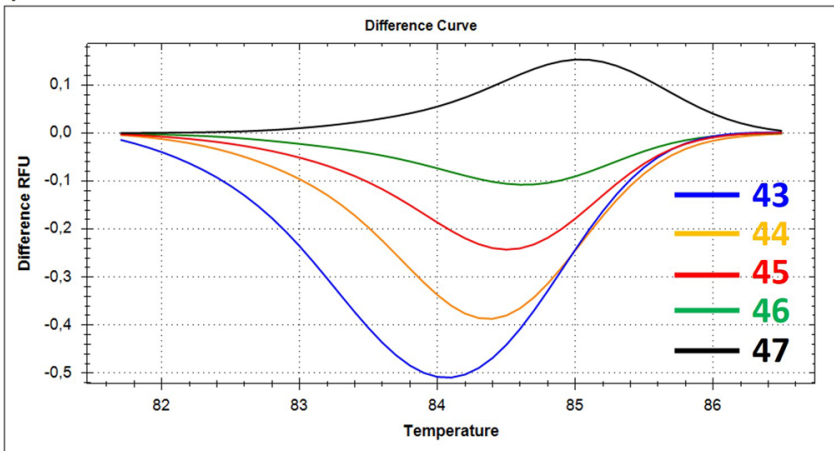

Difference Curve

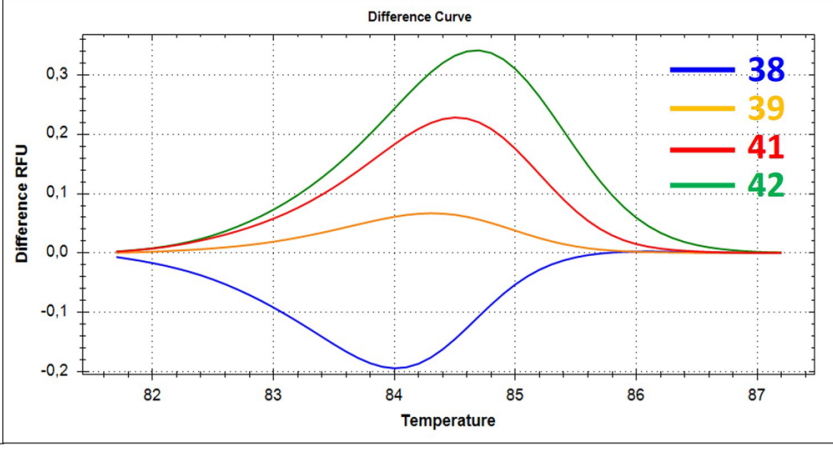

Figure 2. Difference graph for each of six mini-MLST loci. The difference curves are labeled with the GC content in corresponding mini-MLST loci. For locus adk288-368, four $(44,45,46,47)$ out of eight predicted alleles are displayed. For locus fumC227-390, four $(58,61,62,63)$ out of 14 predicted alleles are displayed. For locus gyrB147-191, four $(24,25,26,27)$ out of seven predicted alleles are displayed. For locus icd144-218, four $(43,44,45,47)$ out of eight predicted alleles are displayed. For locus purA254-334, five $(43,44,45,46,47)$ out of seven predicted alleles are displayed. For locus recA82-143, four $(38,39,40,41)$ out of 10 predicted alleles are displayed.

were divided into $45 \mathrm{STs}$ and $34 \mathrm{MelTs}$, respectively. The mini-MST's discriminatory power in our group was $\mathrm{D}=0.9231$ (CI 95\% 0.8958-0.9504) against MLST’s D =0.9364 (CI 95\% 0.9077-0.9652) with the inter-rater agreement $\kappa=0.8979(\mathrm{SD} \pm 0.0091)$.

In silico analysis of 73,704 strains retrieved from EnteroBase ${ }^{15}$ was performed to validate the mini-MLST typing application on large sample sets. The discriminatory power on this substantial set of samples was $\mathrm{D}=0.9465$ (CI 95\% 0.9726-0.9736) for mini-MLST and D=0.9731 (CI 95\% 0.9726-0.9736) for MLST.

Mini-MLST as a tool for outbreak investigation. An increased incidence in extended-spectrum $\beta$-lactamases (ESBL) E. coli was observed in March and May 2016 at the Neonatal Department (UHB). In total, 15 ESBL E. coli isolates were isolated from blood cultures, rectal swabs, and neonates' urine. Environment swabs (room, baths, incubators), health-worker swabs and breast milk samples (sterilized prior to administration to the neonate) were also tested. A single ESBL E. coli isolate was recovered from breast milk and none from the environmental and health-workers swabs. All isolates were subjected to molecular typing analysis using repPCR, MLST and mini-MLST. As a control, four ESBL E. coli urine isolates isolated from different departments at the UHB were added to our analyses. The isolates were differentiated into 4 rep-profiles, 4 STs and 4 MelTs 


\begin{tabular}{|c|c|c|c|c|}
\hline Mini-MLST loci & Allele (GC number) & $\operatorname{Tm} 95 \% \mathrm{CI}\left({ }^{\circ} \mathrm{C}\right)$ & Tm absolute range $\left({ }^{\circ} \mathrm{C}\right)$ & Distribution of captured alleles \\
\hline \multirow{4}{*}{ adk288-368 } & 44 & $82.61-82.73$ & $82.6-82.8$ & 20 \\
\hline & 45 & $83.26-83.39$ & $83.2-83.4$ & 15 \\
\hline & 46 & $83.56-83.68$ & $83.5-83.8$ & 125 \\
\hline & 47 & $84.28-84.37$ & $84.3-84.4$ & 5 \\
\hline \multirow{4}{*}{ fumC227-390 } & 58 & $83.29-83.39$ & $83.3-83.4$ & 8 \\
\hline & 61 & $84.33-84.48$ & $84.3-84.5$ & 18 \\
\hline & 62 & $84.57-84.66$ & $84.5-84.7$ & 73 \\
\hline & 63 & $84.87-84.99$ & $84.8-85.1$ & 66 \\
\hline \multirow{4}{*}{ gyrB147-191 } & 24 & $81.63-81.74$ & $81.6-81.8$ & 50 \\
\hline & 25 & $82.20-82.31$ & $82.2-82.4$ & 67 \\
\hline & 26 & $82.58-82.67$ & $82.6-82.7$ & 19 \\
\hline & 27 & $82.94-83.07$ & $82.9-83.1$ & 29 \\
\hline \multirow{4}{*}{ icd144-218 } & 43 & $83.60-83.76$ & $83.5-83.8$ & 41 \\
\hline & 44 & $84.04-84.19$ & $84.0-84.2$ & 33 \\
\hline & 45 & $84.49-84.61$ & $84.4-84.7$ & 87 \\
\hline & 47 & NA & 85.3 & 1 \\
\hline \multirow{5}{*}{ purA254-334 } & 43 & NA & 83.9 & 1 \\
\hline & 44 & $84.19-84.38$ & $84.2-84.5$ & 50 \\
\hline & 45 & $84.65-84.81$ & $84.6-84.7$ & 49 \\
\hline & 46 & $84.91-85.02$ & $84.7-84.9$ & 64 \\
\hline & 47 & NA & 85.3 & 1 \\
\hline \multirow{4}{*}{ recA82-143 } & 38 & NA & 83.8 & 1 \\
\hline & 39 & $84.23-84.41$ & $84.1-84.5$ & 63 \\
\hline & 40 & $84.65-84.81$ & $84.6-84.9$ & 46 \\
\hline & 41 & $85.14-85.28$ & $85.1-85.3$ & 55 \\
\hline
\end{tabular}

Table 2. Confidence interval 95\% (95\% CI) of melting temperature (Tm), Tm absolute range and HRM normalization regions for obtained mini-MLST loci.

(Fig. 3) indicating the concordance between methods was $100 \%$, whereas no possible higher discriminatory power could be obtained from any of the used methods. Based on typing, the breast milk isolate was identical to the isolates recovered from the neonates. As a result, an immediate sterilizer inspection was performed, and crucial technical damage resulting in its impaired function was discovered. After replacing the sterilizer, no further ESBL E. coli cases in breast milk were observed.

WGS typing, MLST, rep-PCR and mini-MLST concordance. The concordance of WGS, MLST, rep-PCR and mini-MLST and a comparison of their discriminatory power were tested on a subset of isolates obtained during a local epidemiological study conducted at the Department of Internal Medicine-Hematology and Oncology, UHB between 5/2019 and 7/2019. In total, 21 ESBL E. coli isolates were obtained from 14 patients. The 21 isolates were differentiated into 14 WGS clusters (cut-off for clustering isolates together was set to 10 allele differences in a total of 4,637 analyzed genes), 11 STs and 11 MelTs and 12 rep-profiles (Fig. 4). While the ST and mini-MLST results were in exact concordance, the rep-PCR and WGS data analysis divided samples belonging to ST58 into two different clusters. In addition, the WGS further sorted ST131 into three WGS clusters against one MelT and one rep-profile.

\section{Discussion}

MLST and PFGE are still considered the gold standards for molecular typing. However, the development and application of WGS-based techniques is rising, along with a reduction in their costs. WGS has an unsurpassed discriminatory power over other typing methods but is also the most demanding approach in terms of difficulty and data analysis. In contrast, HRM-based methods are extremely cheap, fast and at the same time also very robust and easily portable between laboratories. HRM has already been effectively used to detect and identify antimicrobial resistance, to screen and identify target mutations, evaluate bacterial population structure and genetic diversity ${ }^{4}$. Mini-MLST typing schemes have already been successfully validated for K. pneumoniae ${ }^{5}, S$. aureus $^{6}$, E. faecium ${ }^{7}$ and S. pyogenes ${ }^{8}$. Regarding E.coli, apart from a number of methods designated for species identification, use of HRM methods has been increasing over time and used to quantify virulence and resistance genes or are currently available ${ }^{9-11}$. In terms of molecular typing, there are two methods available. The first one is designed to identify ST131 as an internationally spread high-risk clone ${ }^{16}$. This method is only capable of distinguishing ST131 from non-ST131 strains, which is not sufficient in most cases. The second method is based on ligation-mediated real-time PCR followed by HRM ${ }^{12}$. While retaining the advantages of HRM (speed, cost, low labor intensity), the main disadvantage of this method remains the reproducibility and transferability due to the lack of support within a globally recognized scheme (e.g., MLST). Although this method may be used for 


\begin{tabular}{|c|c|c|c|}
\hline Melt & ST & Clonal complex & No. of isolates \\
\hline 71 & 93 & ST168 cplx & 2 \\
\hline 76 & 48 & ST10 cplx & 1 \\
\hline 156 & 590 & ST590 cplx & 2 \\
\hline 160 & 88 & ST23 cplx & 1 \\
\hline 166 & 410 & ST23 cplx & 1 \\
\hline 175 & 1049 & ST155 cplx & 2 \\
\hline \multirow{4}{*}{198} & 58 & ST155 cplx & 5 \\
\hline & 399 & ST399 cplx & 1 \\
\hline & 1423 & None & 1 \\
\hline & 1429 & None & 2 \\
\hline 234 & 297 & None & 1 \\
\hline 359 & 684 & ST648 cplx & 1 \\
\hline 380 & 1851 & None & 1 \\
\hline 387 & 69 & ST69 cplx & 5 \\
\hline \multirow{2}{*}{447} & 609 & ST46 cplx & 1 \\
\hline & 6740 & None & 1 \\
\hline 508 & 11,601 & None & 1 \\
\hline \multirow{2}{*}{511} & 43 & ST9 cplx & 1 \\
\hline & 617 & ST101 cplx & 1 \\
\hline 512 & 44 & ST10 cplx & 1 \\
\hline 592 & 117 & None & 1 \\
\hline 623 & 961 & ST12 cplx & 1 \\
\hline 626 & 12 & ST12 cplx & 2 \\
\hline 644 & 141 & None & 3 \\
\hline 653 & 131 & ST131 cplx & 23 \\
\hline 669 & 38 & ST38 cplx & 3 \\
\hline \multirow{2}{*}{696} & 100 & ST100 cplx & 3 \\
\hline & 101 & ST101 cplx & 2 \\
\hline 731 & 80 & ST80 cplx & 1 \\
\hline 736 & 1618 & ST73 cplx & 1 \\
\hline \multirow{2}{*}{737} & 73 & ST73 cplx & 11 \\
\hline & 638 & ST73 cplx & 1 \\
\hline \multirow{3}{*}{738} & 95 & ST95 cplx & 7 \\
\hline & 127 & None & 2 \\
\hline & 5484 & ST95 cplx & 1 \\
\hline 740 & 144 & None & 1 \\
\hline 744 & 429 & ST95 cplx & 1 \\
\hline 756 & 1411 & ST14 cplx & 1 \\
\hline \multirow{2}{*}{761} & 14 & ST14 cplx & 2 \\
\hline & 404 & ST14 cplx & 6 \\
\hline 900 & 2 & None & 1 \\
\hline \multirow{2}{*}{923} & 405 & ST405 cplx & 1 \\
\hline & 406 & ST406 cplx & 1 \\
\hline 924 & 517 & ST469 cplx & 1 \\
\hline 968 & 973 & None & 1 \\
\hline
\end{tabular}

Table 3. mini-MLST and MLST genotyping results of 110 selected E. coli isolates.

direct isolate comparison during a local outbreak investigation, it is not suitable for comparing of large sample sets, long-term studies, and inter-laboratory studies.

The mini-MLST approach is based solely on the GC content of the target locus. Therefore, different sequences with the same GC content are practically indistinguishable using HRM analysis alone. Being specific, this means that hundreds of MLST alleles are converted into just a few mini-MLST alleles (typically 3-10), which is reflected in the lower mini-MLST against MLST discriminatory power. However, the major advantages of mini-MLST are cost-effectiveness, rapid performance, robustness, and great reproducibility accompanied with lower analytical complexity, resulting in straightforward interpretation (Table 4$)^{2,17,18}$. The total price per isolate is approximately $\$ 5$ which is significantly more cost effective than the majority of other typing methods (e.g., $\$ 50$ per complete MLST, $\$ 150$ per WGS). The whole analysis including results evaluation takes about $2.5 \mathrm{~h}$ (excluding 


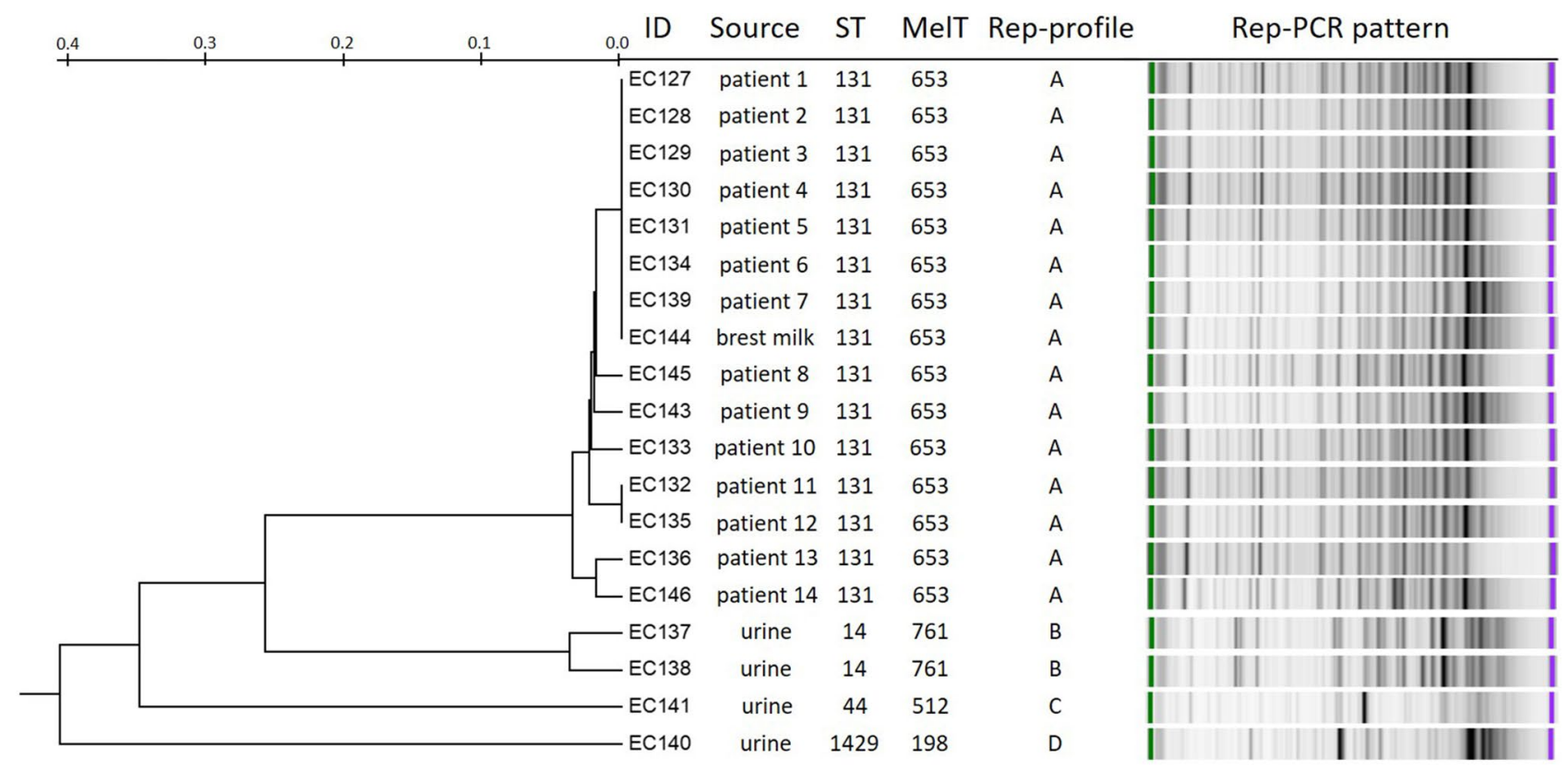

Figure 3. Concordance of rep-PCR, MLST and mini-MLST. Methods were compared with an ESBL E. coli isolate subset collected during a local outbreak at the Neonatal Department (UHB). Rep-profile are based on $95 \%$ similarity.

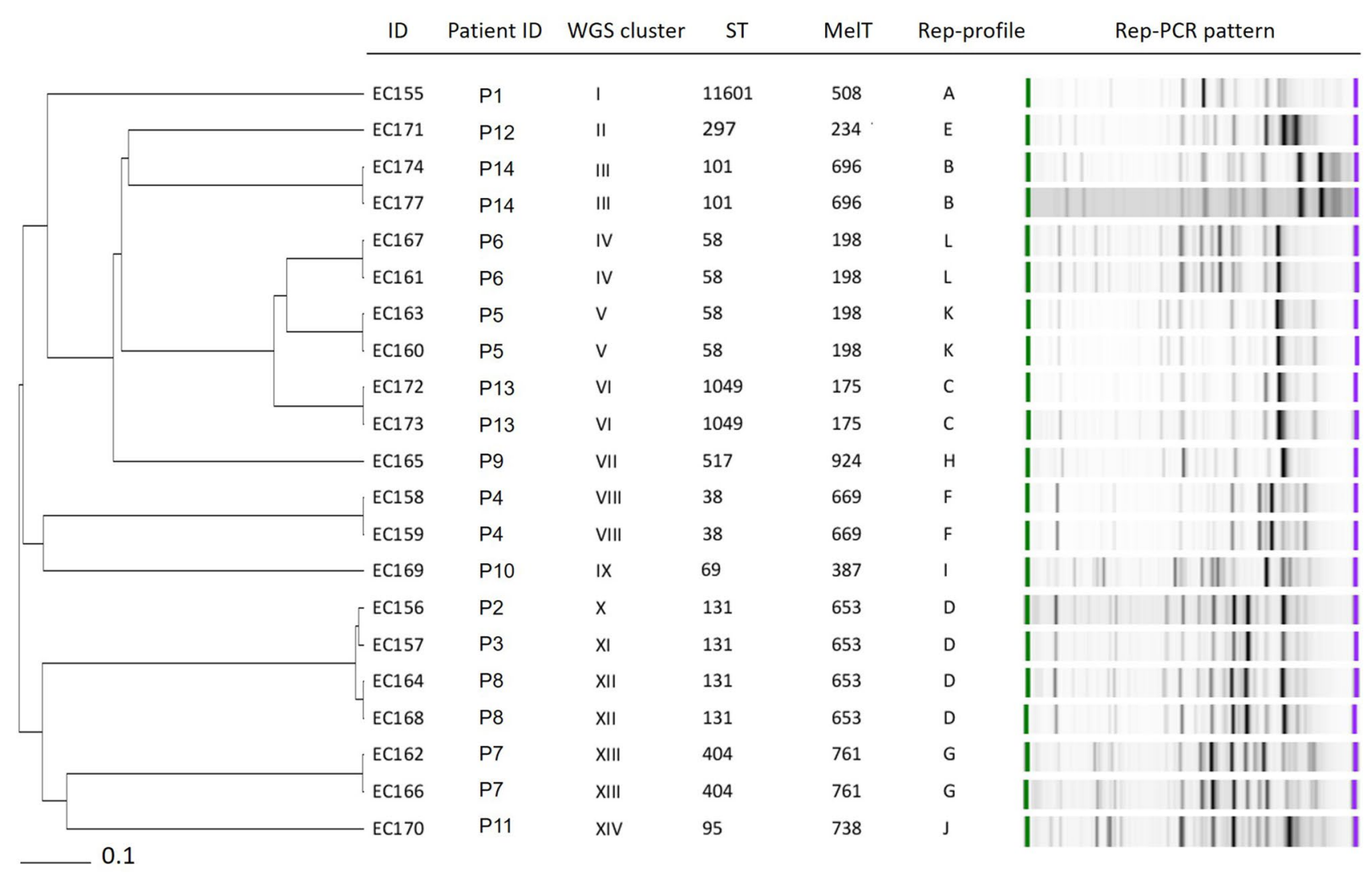

Figure 4. Concordance of wgMLST, MLST, mini-MLST and rep-PCR. Comparison is shown with a subset of 21 ESBL E. coli isolates collected between 5/2019 and 7/2019 at the Department of Internal MedicineHematology and Oncology, University Hospital Brno. The wgMLST clusters were defined by a maximum of 10 allele differences in a total of 4,637 analyzed genes. 


\begin{tabular}{|l|l|l|l|l|}
\hline & Mini-MLST & MLST & Rep-PCR & WGS \\
\hline Discriminatory power & ++ & +++ & ++ & +++++ \\
\hline Typability & +++ & ++++ & +++ & +++++ \\
\hline Reproducibility & ++++ & ++++ & ++ & ++++ \\
\hline Throughput & +++++ & ++ & ++++ & ++ \\
\hline Labor Intensity & + & +++ & ++ & ++++ \\
\hline Analytical Complexity & + & + & + & +++++ \\
\hline Cost & $\$ 5$ & $\$ 50$ & $\$ 10$ & $\$ 150$ \\
\hline Time required & + & +++ & ++ & +++++ \\
\hline
\end{tabular}

Table 4. Comparison of main Mini-MLST, MLST, Rep-PCR and WGS typing characteristics ${ }^{2,17,18}$. Individual characteristics are ranged from + (lowest) to +++++ (highest).

DNA isolation). Moreover, during this study we proved that it is possible to optimize the reaction mixture and temperature to be identical for all existing mini-MLST schemes. This is a considerable advantage as it allows our laboratory to simultaneously type up to 48 isolates of different bacterial species (using 384-well plates). Considering mini-MLST's portability, using our approach may accelerate typing in other laboratories, which is particularly suitable for larger laboratories with a significant number of isolates to be analyzed. Mini-MLST's robustness is based on unique HRM curves produced by fragments with different CG content while using an optimal fragment length (70-200 bp). For longer fragments, the impact of GC content differences on Tm may be reduced and the HRM curves may not be clearly distinguishable ${ }^{6}$. Since mini-MLST is derived from globally recognized MLST, it has great portability and together with its high reproducibility, can be globally implemented into laboratories without substantial effort.

To compare the routinely used MLST and to validate mini-MLST for typing large sample sets, we performed an in silico analysis on 73,704 strains retrieved from EnteroBase database ${ }^{15}$. With a D value of 0.9465 (CI $95 \%$ 0.9726-0.9736) for mini-MLST, a D value of 0.9731 (CI 95\% 0.9726-0.9736) for MLST, mini-MLST proved to have a comparable discrimination power to MLST and to be a suitable method with sufficient discriminatory power for large population studies and long-term screening. Mini-MLST's validation in routine clinical practice was performed against rep-PCR, MLST and WGS on clinical isolates collected during the local outbreak and surveillance study at the UHB. During the local epidemiological study at the Department of Internal Medicine, all typing methods were compared not only with each other but also evaluated against WGS as it provides the highest currently achievable discriminatory potential. This comparison resulted 11 MelTs and 11 ST, 12 repprofiles and 14 WGS clusters. Overall, proposed mini-MLST typing scheme showed great correlation with all three aforementioned methods which was further accompanied by the essential advantages mentioned above. In this case, however, a limited number of isolates need to be taken into account.

Due to the expanding use of next-generation sequencing (incl. WGS), new alleles and/or their combinations (new STs) are being discovered almost daily. To be able to type strains from the newest STs, the conversion key is updated monthly. The current version of the conversion key is available at http://www.cmbgt.cz/mini-mlst/t6353.

From the total number of 10,946 STs known as of $3 / 2021$, only 39 STs are marked as MelT0 i.e., with no specified MelT. In most cases, this is caused by the absence of a specific allele in the source MLST database. In this case, we cannot predict the mini-MLST allele (number of GC bases) and thus determine the specific MelT. Even though the conversion key contains an indication of the missing allele (marked as - 3 error in the conversion key) and the result is MelT0, the HRM curves still can be obtained. Thus, MelT0 does not necessarily mean it is impossible to type. If the particular allele was added to the source data, the change would be reflected in the conversion key after the update.

Mini-MLST can be used not only to resolve an acute epidemiological situation as a rapid typing method, but also for prospective monitoring of high-risk bacterial strains' occurrence. This is possible due to the correlation between the biological properties and the ST/genotype, previously described for E. coli high-risk clones belonging to ST38 (MelT669), ST69 (MelT387), ST73 (MelT737), ST95 (MelT738), ST131 (MelT653), ST155 (MelT175), ST393 (MelT343), ST405 (MelT923), ST410 (MelT166) and ST648 (MelT359) ${ }^{19-21}$. At the same time, the better we know the local bacterial population structure and its properties, the better we can respond to the occurrence of new or emergent virulent and/or multidrug-resistant strains. A two-step approach using miniMLST can be advantageously used for both prospective surveillance and retrospective molecular typing. Our results clearly showed that the isolates distinguished by mini-MLST are similarly distinguished by WGS-based typing. Therefore, only isolates from the same MelT should be processed to the next typing step during an outbreak investigation involving WGS. This will allow hospitals to concentrate focus and resources specifically on the outbreak strains and their subsequent in-depth typing. On the other hand, in studies characterizing a large bacterial population, it is advantageous both in terms of time and cost to select strains from different MelTs as it prevents sequencing identical strains and acquiring redundant information.

To conclude, mini-MLST has great portability, low labor intensity, great cost-efficiency and very high throughput, which represents immense benefits, even when those are accompanied with its slightly lower discriminatory power than other typing methods. Our results proved mini-MLST is a great method for rapid and cost-effective screening and subgrouping for large isolates sets and/or quick strain typing during outbreaks. In addition, it is also suitable for prospective surveillance monitoring of emergent and high-risk E. coli clones. 


\section{Material and methods}

Target identification and primer design. The E. coli MLST scheme for 10,946 sequence types (STs) (data to 31/1/2021), including allele sequences for $a d k$, fum , $g y r B, i c d, m d h$, purA and $r e c A$ genes were downloaded from the EnteroBase website (http://enterobase.warwick.ac.uk/species/ecoli/download_7_gene) ${ }^{13}$. All genes were concatenated and aligned using MEGA7 software ${ }^{22}$.

The SNPs that change the percentage content of $\mathrm{G}+\mathrm{C}$ were identified and selected for further analysis as the $\mathrm{A} \leftrightarrow \mathrm{T}$ and $\mathrm{C} \leftrightarrow \mathrm{G}$ nucleotide changes cannot be generally/commonly detected using HRM analysis. The $m d h$ genes were excluded from further analyses as no significant SNPs were found within this gene.

The primer sets were designed using Primer3 v 0.4.0 (http://bioinfo.ut.ee/primer3-0.4.0/) and targeted conserved regions flanking previously identified SNPs (Table 1). From the available literature, we determined the optimal amplicon length for HRM analysis ranged between 50 to $200 \mathrm{bp}^{5-8}$.

Mini-MLST scheme design. Predicting the HRM curve and assigning the melting type (MelT) were carried out using our in-house MELT2MELT software. Each analyzed locus sequence was processed as follows. Specific forward and reverse primers for all loci were found in amplicons using a simple regex search. The number of $\mathrm{G}$ and $\mathrm{C}$ bases between a pair of primers in every gene in the selected sequence was counted and stored in a table containing all analyzed sequence types. The order of the rows in the table was rearranged according to the increasing number of $\mathrm{G}$ and $\mathrm{C}$ bases in every analyzed gene. Finally, a MelT number was assigned to each ST by assigning a number one to the first row of the table and increasing this number by one for every ST whose G and $\mathrm{C}$ base numbers were different from the previous ST.

Discriminatory power calculation. To determine the discriminatory power of proposed mini-MLST scheme and MLST, Simpson's Index of Diversity (D) ${ }^{23}$ was calculated using a freely accessible MATLAB (MathWorks, USA) code available at http://www.comparingpartitions.info/.

Clinical Isolates. For mini-MLST validation, a total of 169 clinical E. coli isolates collected between 3/2016 and 7/2019 at the Department of Clinical microbiology (UHB) were used. All isolates were identified using Matrix-Assisted Laser Desorption/Ionization Time-of-Flight Mass Spectrometry (MALDI-TOF). Pure culture colonies were harvested and resuspended in $2 \mathrm{ml}$ of sterile water (density corresponding to 5.0 McFarland standard) and stored at $-20^{\circ} \mathrm{C}$ for further molecular analyses. All isolates were stored in cryotubes ITEST Kryobanka B (ITEST plus, Czech Republic). Out of all 169 E. coli isolates, 19 were collected during the local outbreak at the Neonatal Department, UHB in 3/2016-5/2016 and 21 isolates were collected during a surveillance study conducted at the Department of Hematology and Oncology (UHB) in 5/2019-7/2019.

Sample set for in silico analysis. To validate the mini-MLST on a large sample set, the MLST data from 100,000 most recently uploaded E. coli strains were retrieved (http://enterobase.warwick.ac.uk/species/ecoli/ search_strains). Out of 100,000 strains, only strains with specified ST $(n=73,704)$ were used to compare in silico mini-MLST and MLST. The complete list of all 73,704 strains is listed in the Supplementary Table S1.

DNA isolation. Genomic DNA (gDNA) was isolated using Chelex 100 Resin (Bio-Rad, USA). The overnight bacterial cultures were homogenized in $100 \mu \mathrm{L}$ of $5 \% \mathrm{w} / \mathrm{v}$ Chelex 100 Resin with vortex. The obtained suspensions were incubated for $10 \mathrm{~min}$ at $100^{\circ} \mathrm{C}$ and then centrifuged for $2 \mathrm{~min}$ at $15,500 \mathrm{rcf}$. Each strain's supernatant containing gDNA was transferred into a clean microtube. For WGS, gDNA was purified using GenElute Bacterial Genomic DNA Kit (SIGMA-ALDRICH, USA).

Mini-MLST. Mini-MLST was performed on a Bio-Rad CFX96 platform (Bio-Rad, USA). The reaction mixture contained $10 \mu \mathrm{L} 2 \times$ SensiFAST HRM mix (Meridian Bioscience, UK), $0.4 \mu \mathrm{M}$ of each primer, $1 \mu \mathrm{L}$ of gDNA $(30 \mathrm{ng} / \mu \mathrm{L})$ and deionized water to a final volume of $20 \mu \mathrm{L}$. Thermo cycling parameters were: $95^{\circ} \mathrm{C}$ for $3 \mathrm{~min}, 40$ cycles of $95^{\circ} \mathrm{C}$ for $5 \mathrm{~s}, 65^{\circ} \mathrm{C}$ for $10 \mathrm{~s}$ and $72^{\circ} \mathrm{C}$ for $20 \mathrm{~s}$, followed by one cycle of $95^{\circ} \mathrm{C}$ for $2 \mathrm{~min}$ and $50^{\circ} \mathrm{C}$ for $20 \mathrm{~s}$, terminated by HRM ramping from 72 to $88^{\circ} \mathrm{C}$, increasing by $0.1^{\circ} \mathrm{C}$ at each step. The results were interpreted using the current version of our conversion key, which is available for free download at http://www.cmbgt.cz/ mini-mlst/t6353. The conversion key is regularly updated on a monthly basis.

Multilocus sequence typing. In total, 110 strains were selected for a full MLST sequencing scheme according to the protocol described by Wirth et al. ${ }^{15}$. The current version of the E. coli MSLT database is available on http://enterobase.warwick.ac.uk/species/ecoli/download_7_gene.

Rep-PCR. To generate DNA fingerprint patterns, Rep-PCR primers REP1R and REP2I were used ${ }^{24}$, following the protocols described previously ${ }^{25}$. The PCR amplicons were analyzed using Agilent 2100 Bioanalyzer (Agilent Technologies, USA) and the previously described algorithm was used ${ }^{26}$ to determine the rep-profiles.

Whole genome sequencing. Sequencing libraries were prepared using KAPA HyperPlus Kits (Roche, Switzerland). Sequencing was performed on the Illumina MiSeq platform using the MiSeq Reagent Kit v2 (500-cycles) (Illumina, USA). After quality control, the reads were trimmed via Trimmomatic ${ }^{27}$. BurrowsWheeler Alignment MEM (v0.7.17-r1188) ${ }^{28}$ was used for reference-based assembly. E. coli NC_002695.2 was used as a reference genome. Samtools ${ }^{29}$ was used to remove low-quality and duplicated reads, followed by con- 
sensus sequence generation. The assembled genomes were analyzed using Ridom SeqSphere+(Ridom GmbH, $\mathrm{DE})$.

\section{Data availability}

All E. coli raw illumina sequence data from this study have been deposited in the Sequence Read Archive under the BioProject No. PRJNA695195. All MLST FASTA files are available from https://github.com/tysek3/ ESCO-mini-MLST-Sup-files.git. The MelT conversion key is available to download from http://www.cmbgt.cz/ mini-mlst/t6353.

Received: 16 March 2021; Accepted: 27 July 2021

Published online: 16 August 2021

\section{References}

1. Vila, J. et al. Escherichia coli: An old friend with new tidings. FEMS Microbiol. Rev. 40, 437-463. https://doi.org/10.1093/femsre/ fuw005 (2016).

2. MacCannell, D. Bacterial strain typing. Clin. Lab. Med. 33, 629-650. https://doi.org/10.1016/j.cll.2013.03.005 (2013).

3. Enright, M. C. \& Spratt, B. G. Multilocus sequence typing. Trends Microbiol. 7, 482-487. https://doi.org/10.1016/s0966-842x(99) 01609-1 (1999).

4. Tamburro, M. \& Ripabelli, G. High Resolution Melting as a rapid, reliable, accurate and cost-effective emerging tool for genotyping pathogenic bacteria and enhancing molecular epidemiological surveillance: A comprehensive review of the literature. Ann. Ig. 29, 293-316. https://doi.org/10.7416/ai.2017.2153 (2017).

5. Andersson, P., Tong, S. Y., Bell, J. M., Turnidge, J. D. \& Giffard, P. M. Minim typing: A rapid and low cost MLST based typing tool for Klebsiella pneumoniae. PLoS ONE 7, e33530. https://doi.org/10.1371/journal.pone.0033530 (2012).

6. Lilliebridge, R. A., Tong, S. Y., Giffard, P. M. \& Holt, D. C. The utility of high-resolution melting analysis of SNP nucleated PCR amplicons: An MLST based Staphylococcus aureus typing scheme. PLoS ONE 6, e19749. https://doi.org/10.1371/journal.pone. 0019749 (2011).

7. Tong, S. Y. et al. High-resolution melting genotyping of Enterococcus faecium based on multilocus sequence typing derived single nucleotide polymorphisms. PLoS ONE 6, e29189. https://doi.org/10.1371/journal.pone.0029189 (2011).

8. Richardson, L. J. et al. Preliminary validation of a novel high-resolution melt-based typing method based on the multilocus sequence typing scheme of Streptococcus pyogenes. Clin. Microbiol. Infect. 17, 1426-1434. https://doi.org/10.1111/j.1469-0691. 2010.03433.x (2011).

9. Bender, A. C., Faulkner, J. A., Tulimieri, K., Boise, T. H. \& Elkins, K. M. High resolution melt assays to detect and identify. Microorganisms https://doi.org/10.3390/microorganisms8040561 (2020).

10. Wang, W., Zijlstra, R. T. \& Gänzle, M. G. Identification and quantification of virulence factors of enterotoxigenic Escherichia coli by high-resolution melting curve quantitative PCR. BMC Microbiol. 17, 114. https://doi.org/10.1186/s12866-017-1023-5 (2017).

11. Edwards, T. et al. A highly multiplexed melt-curve assay for detecting the most prevalent carbapenemase, ESBL, and AmpC genes. Diagn. Microbiol. Infect. Dis. 97, 115076. https://doi.org/10.1016/j.diagmicrobio.2020.115076 (2020).

12. Woksepp, H. et al. High-resolution melting-curve analysis of ligation-mediated real-time PCR for rapid evaluation of an epidemiological outbreak of extended-spectrum-beta-lactamase-producing Escherichia coli. J. Clin. Microbiol. 49, 4032-4039. https:// doi.org/10.1128/JCM.01042-11 (2011).

13. Zhou, Z. et al. The EnteroBase user's guide, with case studies on. Genome Res. 30, 138-152. https://doi.org/10.1101/gr.251678.119 (2020).

14. Price, E. P., Inman-Bamber, J., Thiruvenkataswamy, V., Huygens, F. \& Giffard, P. M. Computer-aided identification of polymorphism sets diagnostic for groups of bacterial and viral genetic variants. BMC Bioinform. 8, 278. https://doi.org/10.1186/1471-2105-8-278 (2007).

15. Wirth, T. et al. Sex and virulence in Escherichia coli: An evolutionary perspective. Mol. Microbiol. 60, 1136-1151. https://doi.org/ 10.1111/j.1365-2958.2006.05172.x (2006).

16. Harrison, L. B. \& Hanson, N. D. High-resolution melting analysis for rapid detection of sequence type 131 Escherichia coli. Antimicrob. Agents Chemother. 61, 4. https://doi.org/10.1128/AAC.00265-17 (2017).

17. Sabat, A. J. et al. Overview of molecular typing methods for outbreak detection and epidemiological surveillance. Euro Surveill. 18, 20380. https://doi.org/10.2807/ese.18.04.20380-en (2013).

18. Ranjbar, R., Karami, A., Farshad, S., Giammanco, G. M. \& Mammina, C. Typing methods used in the molecular epidemiology of microbial pathogens: A how-to guide. New Microbiol. 37, 1-15 (2014).

19. Mathers, A. J., Peirano, G. \& Pitout, J. D. The role of epidemic resistance plasmids and international high-risk clones in the spread of multidrug-resistant Enterobacteriaceae. Clin. Microbiol. Rev. 28, 565-591. https://doi.org/10.1128/CMR.00116-14 (2015).

20. Doumith, M. et al. Rapid identification of major Escherichia coli sequence types causing urinary tract and bloodstream infections. J. Clin. Microbiol. 53, 160-166. https://doi.org/10.1128/JCM.02562-14 (2015).

21. Roer, L. et al. Sequence type 410 is causing new international high-risk clones. Sphere https://doi.org/10.1128/mSphere.00337-18 (2018).

22. Kumar, S., Stecher, G. \& Tamura, K. MEGA7: Molecular evolutionary genetics analysis version 7.0 for bigger datasets. Mol. Biol. Evol. 33, 1870-1874. https://doi.org/10.1093/molbev/msw054 (2016).

23. Hunter, P. R. \& Gaston, M. A. Numerical index of the discriminatory ability of typing systems: An application of Simpson's index of diversity. J. Clin. Microbiol. 26, 2465-2466. https://doi.org/10.1128/JCM.26.11.2465-2466.1988 (1988)

24. Versalovic, J., Koeuth, T. \& Lupski, J. R. Distribution of repetitive DNA sequences in eubacteria and application to fingerprinting of bacterial genomes. Nucleic Acids Res. 19, 6823-6831. https://doi.org/10.1093/nar/19.24.6823 (1991).

25. Svec, P., Pantůček, R., Petráš, P., Sedláček, I. \& Nováková, D. Identification of Staphylococcus spp. using (GTG)5-PCR fingerprinting. Syst. Appl. Microbiol. 33, 451-456. https://doi.org/10.1016/j.syapm.2010.09.004 (2010).

26. Skutkova, H., Vitek, M., Bezdicek, M., Brhelova, E. \& Lengerova, M. Advanced DNA fingerprint genotyping based on a model developed from real chip electrophoresis data. J. Adv. Res. 18, 9-18. https://doi.org/10.1016/j.jare.2019.01.005 (2019).

27. Bolger, A. M., Lohse, M. \& Usadel, B. Trimmomatic: A flexible trimmer for Illumina sequence data. Bioinformatics 30, 2114-2120. https://doi.org/10.1093/bioinformatics/btu170 (2014).

28. Li, H. \& Durbin, R. Fast and accurate long-read alignment with Burrows-Wheeler transform. Bioinformatics 26, 589-595. https:// doi.org/10.1093/bioinformatics/btp698 (2010).

29. Li, H. et al. The Sequence Alignment/Map Format and SAMtools. Bioinformatics 25, 2078-2079. https://doi.org/10.1093/bioin formatics/btp352 (2009). 


\section{Acknowledgements}

This study was supported by the Ministry of Health of the Czech Republic, Grant No. NV19-09-00430, all rights reserved; Ministry of Health, Czech Republic - conceptual development of research organization $(\mathrm{FNBr}$, 65269705); and MUNI/A/1595/2020.

\section{Author contributions}

Conceptualization, M.B., M.L.; data curation, M.B., M.N., K.S., H.S.; formal analysis, M.B.; funding, M.L., J.M.; sample collection, I.K.; laboratory experiments, M.B.; S.K.; J.H.; A.K.; supervision, M.B., M.L., J.M; manuscript preparation-original draft, M.B.; review and editing, M.B., M.L., S.K.; review of final manuscript, all authors.

\section{Competing interests}

The authors declare no competing interests.

\section{Additional information}

Supplementary Information The online version contains supplementary material available at https://doi.org/ 10.1038/s41598-021-96148-3.

Correspondence and requests for materials should be addressed to M.L.

Reprints and permissions information is available at www.nature.com/reprints.

Publisher's note Springer Nature remains neutral with regard to jurisdictional claims in published maps and institutional affiliations.

(c) (i) Open Access This article is licensed under a Creative Commons Attribution 4.0 International License, which permits use, sharing, adaptation, distribution and reproduction in any medium or format, as long as you give appropriate credit to the original author(s) and the source, provide a link to the Creative Commons licence, and indicate if changes were made. The images or other third party material in this article are included in the article's Creative Commons licence, unless indicated otherwise in a credit line to the material. If material is not included in the article's Creative Commons licence and your intended use is not permitted by statutory regulation or exceeds the permitted use, you will need to obtain permission directly from the copyright holder. To view a copy of this licence, visit http://creativecommons.org/licenses/by/4.0/.

(C) The Author(s) 2021 